\title{
The hidden genomic diversity of ciliated protists revealed by single-cell genome sequencing
}

\author{
Wenbing Chen ${ }^{1,2+}$, Changling Zuo ${ }^{1 \dagger}$, Chundi Wang ${ }^{3,4}$, Tengteng Zhang ${ }^{3,4}$, Liping Lyu ${ }^{3,4}$, Yu Qiao ${ }^{3,4}$,
} Fangqing Zhao ${ }^{1,2^{*}}$ (iD) and Miao Miao ${ }^{1 *}$

\begin{abstract}
Background: Ciliated protists are a widely distributed, morphologically diverse, and genetically heterogeneous group of unicellular organisms, usually known for containing two types of nuclei: a transcribed polyploid macronucleus involved in gene expression and a silent diploid micronucleus responsible for transmission of genetic material during sexual reproduction and generation of the macronucleus. Although studies in a few species of culturable ciliated protists have revealed the highly dynamic nature of replicative and recombination events relating the micronucleus to the macronucleus, the broader understanding of the genomic diversity of ciliated protists, as well as their phylogenetic relationships and metabolic potential, has been hampered by the inability to culture numerous other species under laboratory conditions, as well as the presence of symbiotic bacteria and microalgae which provide a challenge for current sequencing technologies. Here, we optimized single-cell sequencing methods and associated data analyses, to effectively remove contamination by commensal bacteria, and generated high-quality genomes for a number of Euplotia species.

Results: We obtained eight high-quality Euplotia genomes by using single-cell genome sequencing techniques. The genomes have high genomic completeness, with sizes between 68 and $125 \mathrm{M}$ and gene numbers between $14 \mathrm{~K}$ and $25 \mathrm{~K}$. Through comparative genomic analysis, we found that there are a large number of gene expansion events in Euplotia genomes, and these expansions are closely related to the phenotypic evolution and specific environmental adaptations of individual species. We further found four distinct subgroups in the genus Euplotes, which exhibited considerable genetic distance and relative lack of conserved genomic syntenies. Comparative genomic analyses of Uronychia and its relatives revealed significant gene expansion associated with the ciliary movement machinery, which may be related to the unique and strong swimming ability.
\end{abstract}

Conclusions: We employed single-cell genomics to obtain eight ciliate genomes, characterized the underestimated genomic diversity of Euplotia, and determined the divergence time of representative species in this subclass for the first time. We also further investigated the extensive duplication events associated with speciation and environmental adaptation. This study provides a unique and valuable resource for understanding the evolutionary history and genetic diversity of ciliates.

Keywords: Ciliate, Euplotia, Genetic diversity, Single-cell genomics

\footnotetext{
*Correspondence: zhfq@biols.ac.cn; miaomiao@ucas.ac.cn

${ }^{\dagger}$ Wenbing Chen and Changling Zuo contributed equally to this work.

'Savaid Medical School, University of Chinese Academy of Sciences, Beijing 100049, China

Full list of author information is available at the end of the article
}

(c) The Author(s). 2021 Open Access This article is licensed under a Creative Commons Attribution 4.0 International License, which permits use, sharing, adaptation, distribution and reproduction in any medium or format, as long as you give appropriate credit to the original author(s) and the source, provide a link to the Creative Commons licence, and indicate if changes were made. The images or other third party material in this article are included in the article's Creative Commons licence, unless indicated otherwise in a credit line to the material. If material is not included in the article's Creative Commons licence and your intended use is not permitted by statutory regulation or exceeds the permitted use, you will need to obtain permission directly from the copyright holder. To view a copy of this licence, visit http://creativecommons.org/licenses/by/4.0/ The Creative Commons Public Domain Dedication waiver (http://creativecommons.org/publicdomain/zero/1.0/) applies to the data made available in this article, unless otherwise stated in a credit line to the data. 


\section{Background}

Ciliates, the unicellular organisms with the most complex lifestyles, are found anywhere there is water [1]. Unlike other eukaryotes, ciliates have two types of cell nuclei: silent diploid micronuclei and highly transcribed polyploid macronuclei. The micronucleus carries all the genetic material and is responsible for the transmission of genetic material during sexual reproduction, while the macronucleus genome usually has tens of thousands of copies of the genome and is responsible for the expression of genes [2]. Noncoding RNAs mediate the massive replication and recombination of the micronucleus genome and the addition of telomeres to each chromosome, eventually generating the macronucleus genome [3]. The investigation of this unique process led to the discovery of nucleases and telomeres [3]. However, this phenomenon also poses an obstacle to the study of the ciliate genome. Most ciliates feed on bacteria and microalgae [4], and some ciliates establish transient or stable symbiotic relationships with their prey $[5,6]$. These cohabiting or endosymbiotic bacteria or microalgae pose a great challenge for access to the ciliate genome, especially for nonculturable ciliates.

There have been a large number of gene duplication events in ciliate genomes, bringing their gene numbers close to those of many plants and metazoans [7]. These gene duplication phenomena provide good material to study the genomic diversity and evolution of ciliates. However, due to the lack of complete genomic information, these studies have been conducted in only some model organisms, such as Tetrahymena [8] and Paramecium [7]. There are approximately 27,000-40,000 known species of ciliates [1], but genomic or transcriptomic data available for fewer than 60 species, which greatly limits the knowledge of their genomic diversity.

In recent years, single-cell sequencing technology has been applied to the sequencing of unculturable ciliates, which has helped us to solve controversial problems in ciliate phylogenetic and genomic studies [9, 10]. However, previous single-cell sequencing methods were unable to obtain complete ciliate genomes and had substantial bacterial sequence contamination, which posed a significant challenge for subsequent ciliate genome analysis. In this study, we took full advantage of different single-cell sequencing technologies and efficient genome assembly methods to obtain eight high-quality Euplotia genome sequences with low contamination. Euplotia, which contains hundreds of species, is a highly diverse subclass in Spirotricha (Fig. 1A). Through comparative genomic analyses, we revealed the genomic diversity of ciliate Euplotia, resolved their phylogenetic relationships, and systematically described their genomic features in relation to environmental adaptation.

\section{Results}

\section{De novo sequencing and assembly of eight Euplotia} genomes

Eight Euplotia samples were collected from coastal waters or freshwater tanks in Qingdao and Shenzhen in China. Five of them inhabit seawater, two brackish water and one freshwater (Fig. 1B). Based on morphological identification, they were classified as Certesia quadrinucleata, Diophrys sp., Euplotes cf. woodruffi, Euplotes parawoodruffi, Euplotes weissei, Euplotes woodruffi, Uronychia binucleate, and Uronychia setigera, respectively. These eight species represent four major genera (Certesia, Diophrys, Euplotes, and Uronychia) in the order Euplotida, whose morphological characteristics have been described previously (Fig. 1B) [4, 11-15].

To obtain clean ciliate cells and eliminate potential contamination, we picked and washed ciliate single cells multiple times using glass pipettes. Considering the difficulty of amplifying whole genomes from single cell, we used both the multiple displacement amplification (MDA) [16] and multiple annealing and looping-based amplification cycle (MALBAC) [17] approaches to amplify genomic DNA from single cells. For each method, we performed the amplification experiments twice and only one cell was used in each experiment (Additional file 1: Table S1). We constructed DNA libraries for each amplification sample and sequenced them using the Illumina NovaSeq 6000 platform. After filtering low-quality reads, the sequencing data for each cell were classified using Kraken [18], and we found MALBAC had a lower bacteria contamination (Fig. 1C, D). To remove potential contamination from endosymbiotic bacteria or microalgae, we employed a filtration pipeline (see the "Methods" section) and ultimately discarded $0.07-28 \%$ of the sequencing data. We then assembled the sequence data for each cell separately and mapped the reads back to the assemblies. MDA had a higher read mapping rate (Fig. $1 C)$, whereas MALBAC had a lower amplification bias (Fig. 1E). After merging the filtered data for each species, we finally generated an average of 15 71 Gb data for each species with high sequencing depth $(156 \sim 700 \times)$. To assess the effect of cell number on genome completeness, we combined the assemblies for each species and evaluate the genome completeness using EukCC [19]. The genome completeness significantly increased with the number of cells used until four assemblies were used (Fig. 1F).

After genome assembly and removal of contaminated contigs, we finally obtained eight ciliate macronuclear genomes with 37,973 89,285 contigs and an N50 length of 934 2,543. The genome size $(74 \sim 125 \mathrm{Mb})$ and GC content $(32.0 \% \sim 45.9 \%)$ were similar to those of other macronuclear genomes of Euplotes (Table 1). To evaluate the completeness of the assembled genomes, all 


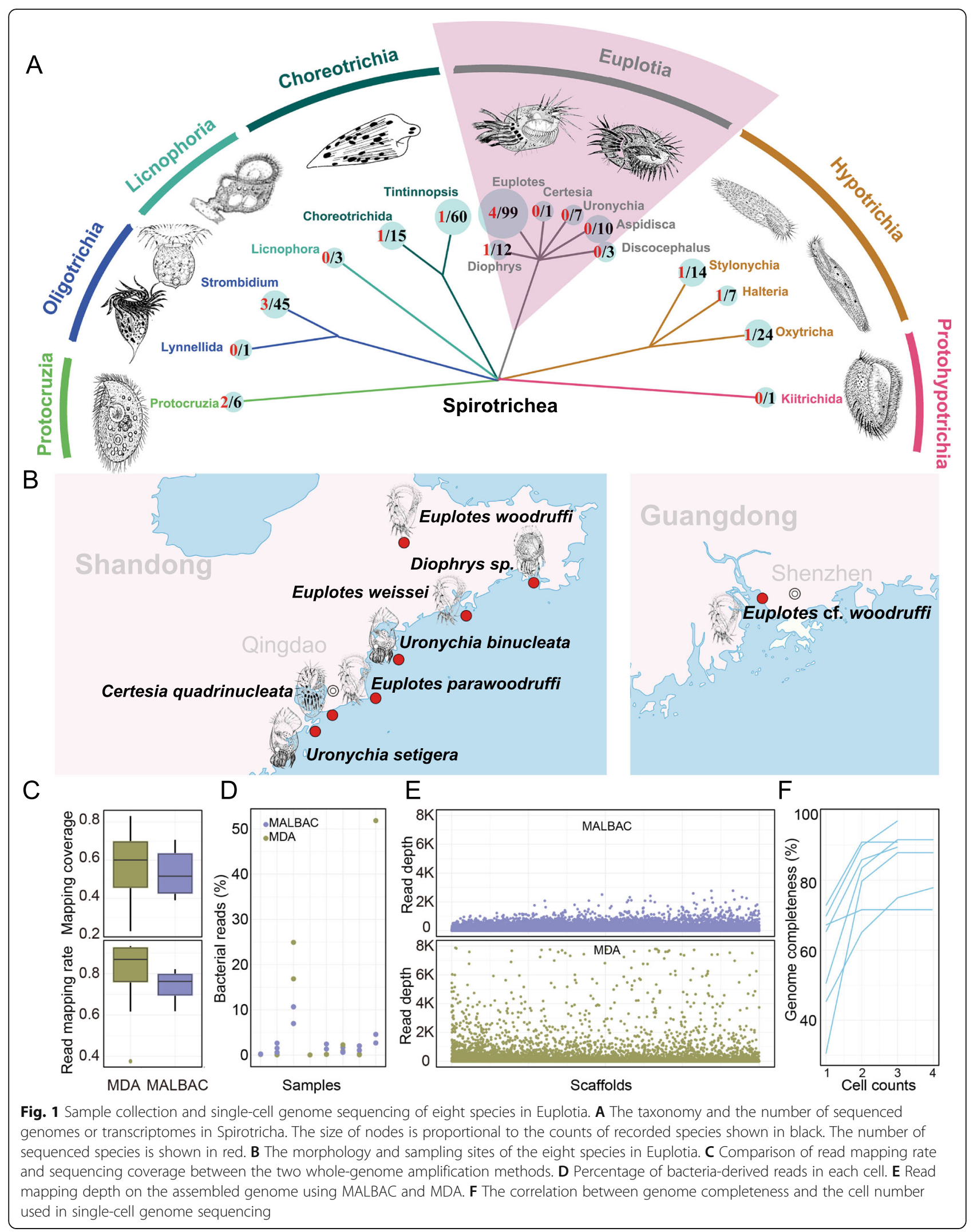


Table 1 Summary of Euplotia genome assembly and annotation

\begin{tabular}{lllllll}
\hline Species & Filtered reads (Gb) & Genome size (bp) & N50 (bp) & GC content (\%) & Genome completeness (\%) & Gene number \\
\hline Certesia quadrinucleata & 59.6 & $74,018,178$ & 1270 & 42.31 & 71.58 & 15,982 \\
Diophrys sp. & 55.1 & $125,778,342$ & 1838 & 36.04 & 90.91 & 25,327 \\
Euplotes cf. woodruffi & 51.4 & $83,224,465$ & 2696 & 36.76 & 96.97 & 14,298 \\
Euplotes focardii & $/$ & $42,117,764$ & 1929 & 32.02 & 95.79 & 9382 \\
Euplotes parawoodruffi & 39.6 & $83,441,685$ & 1610 & 43.02 & 89.47 & 19,635 \\
Euplotes vannus & $/$ & $84,760,489$ & 2692 & 36.89 & 84.85 & 15,078 \\
Euplotes weissei & 66.7 & $84,107,277$ & 934 & 43.86 & 77.89 & 21,295 \\
Euplotes woodruffi & 53.0 & $87,592,728$ & 1887 & 37.72 & 91.58 & 15,830 \\
Moneuplotes crassus & $/$ & $51,565,225$ & 1761 & 36.91 & 95.79 & 11,481 \\
Uronychia binucleata & 15.6 & $68,224,519$ & 2316 & 45.86 & 78.79 & 19,733 \\
Uronychia setigera & 70.9 & $111,284,557$ & 2543 & 36.43 & 87.88 & 20,152 \\
\hline
\end{tabular}

filtered reads were mapped back to their corresponding assemblies. Approximately $94 \%$ of reads could be aligned to the assemblies, and the remaining reads were found to be of bacterial or fungal origin. We further used EukCC [19] and BUSCO [20] to estimate the completeness of the assembled genomes (Additional file 1: Fig. S1) and found that all of them exhibited relatively high completeness ratios, ranging from 71 to $96 \%$, which is comparable to three previously sequenced Euplotes cultured species: 95\% for Euplotes focardii (accession number in NCBI: GCA_001880345.1), 95\% for Moneuplotes crassus (accession number in NCBI: GCA_001880385.1), and $84 \%$ for Euplotes vannus [21] (Table 1). We employed a comprehensive strategy to annotate the genome by combining homolog-based and ab initio approaches. We finally obtained 9382 25,327 proteincoding genes for each species. Most contigs contained $1 \sim 3$ genes, which is consistent to previous studies (Additional file 1: Fig. S2). We found that many genes were assigned to families of closely related genes, which may be derived from gene duplication events. To determine the phylogenetic relationships of the Euplotia and confirm the morphology-based classification, we performed phylogenetic analysis using 157 orthologous genes in all available ciliate genomes using the maximum likelihood (ML) method [22]. The phylogenetic tree showed a similar topology to the phylogenetic analysis based on small subunit rRNA (SSrRNA) reported previously [23, 24]. Uronychia and Diophrys branched earliest in Euplotia and had a closer relationship to each other than to other species. Certesia is sister to Euplotes, forming a monophyletic clade that also includes Moneuplotes crassus (Additional file 1: Fig. S3).

\section{Euplotia is the most diverse subclass in Spirotricha}

To compare the genome characteristics of Euplotia to their relatives in Spirotricha, we further analyzed 14 genomes and 2 transcriptomes of Spirotricha in addition to the eight genomes generated in this study (Additional file 1: Table S2). These 24 species were classified into four subclasses: Euplotia, Hypotrichia, Oligotrichia, and Choreotrichia. After genome annotation, we obtained an average of 17,634 protein-coding genes (Fig. 2A). To evaluate the divergence time of Spirotricha, we performed molecular clock analysis using BEAST [25] with the Tintinnids fossil record time, which was dated back to 580 million years ago (Mya). The time tree showed a similar topology to the ML tree described above (Additional file 1: Fig S3, Fig. 2B). Euplotia may have appeared 1300 Mya after it diverged from Choreotrichia. Uronychia and Diophrys originated from family Uronychiidae and diverged 1100 Mya. In contrast, Hypotrichia, another highly diverse subclass, originated 1000 Mya, significantly later than Euplotia (Fig. 2B). Notably, the divergence time of Euplotia at the species level was earlier than that of Hypotrichia at the genus level.

To further investigate the gene content of these 24 species, we performed homology analysis using OrthoFinder [26] and counted the homologous groups shared between each pair of species. A total of 316,565 genes were clustered into 23,294 homologous groups, of which a large majority (91.7\%) could be assigned to homologous groups; the remaining 17,993 genes were speciesspecific. Euplotia had approximately 3805 homologous groups shared by all 11 species, significantly lower than the number in Hypotrichs (5689 homologous groups) (Fig. 2B). We further obtained single-copy genes through pairwise comparison and performed phylogenetic analysis of these single-copy homologous genes, which revealed the same phylogenetic relationship (Additional file 1: Fig. S4).

To explore the genomic diversity in Euplotia, we clustered the count of genes for each homologous group of Euplotia and Hypotrichia using the k-means algorithm. As shown in Fig. 2C, approximately 4159 homologous groups had no significant gain/loss in any species, and 


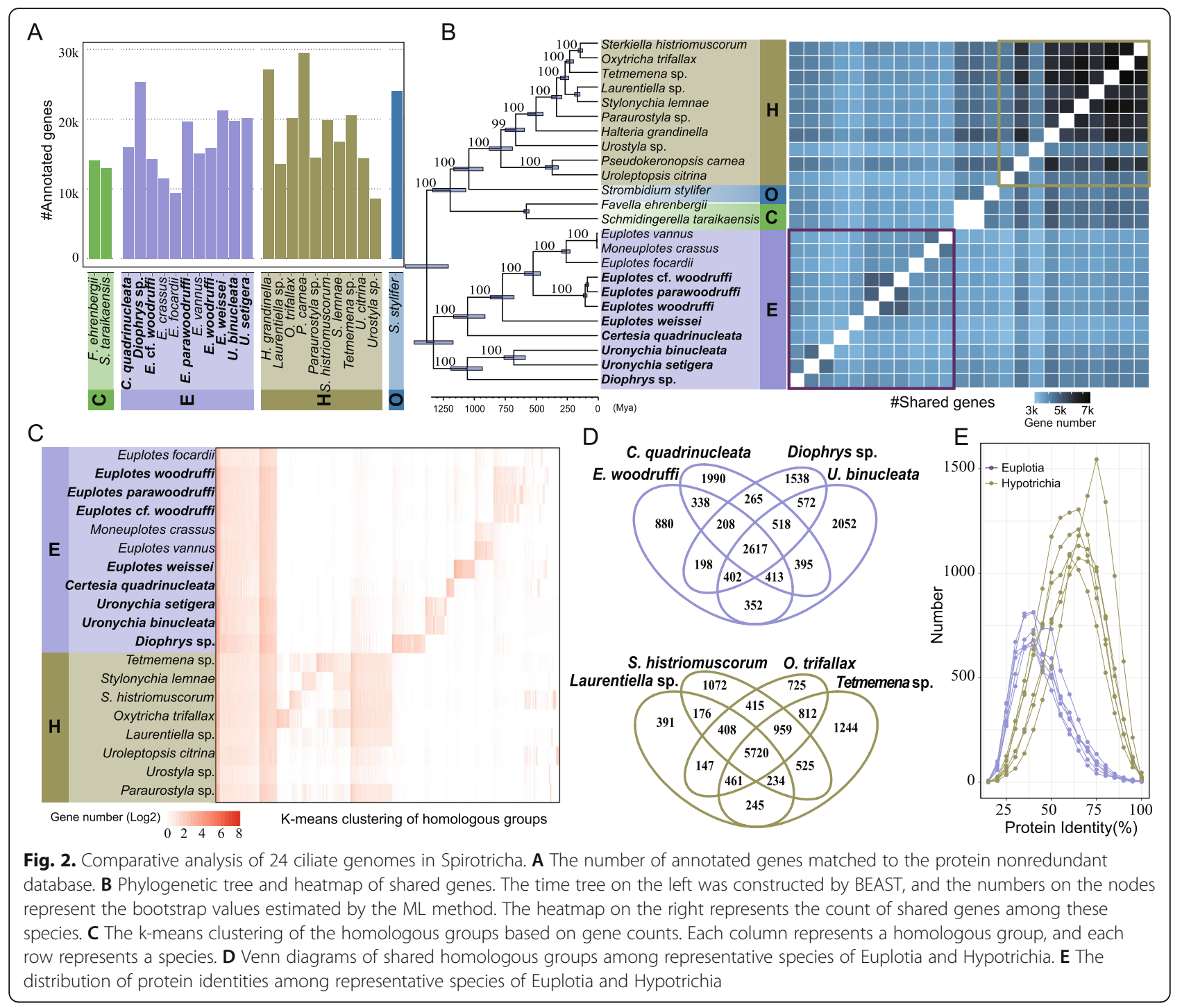

these may represent housekeeping genes in both subclasses. We enriched those genes in Gene Ontology database and found that most of them were involved in functions of catalytic activity, binding activity, metabolic process, or cellular process (Additional file 1: Fig. S5). Hypotrichia had 2822 homologous groups gained in the whole subclass. Euplotia, however, did not show a significant gain cluster at the subclass level; instead, seven clusters of homologous groups were found at the genus or species level. For example, E. vannus and E. crassus were more closely related to each other, sharing 1208 gained homologous groups, whereas E. woodruffi, E. parawoodruffi, and E. cf. woodruffi were clustered together and shared 2087 gained homologous groups. These findings indicated that Euplotia exhibited much higher genetic heterogeneity than Hypotrichia. To further quantify the genetic distances among several species of Euplotia and Hypotrichia, we used four representative species in each subclass and computed the sequence identities of their shared homolog groups (Fig. 2D). The median protein identity within Euplotia was approximately $35 \%$, much lower than that of Hypotrichia (65\%) (Fig. 2E). Taken together, these findings strongly indicate that the genomic diversity in Euplotia has been underestimated.

\section{Extensive gene expansion events in Euplotia}

To explore the gene expansion events of Euplotia genomes, we used both homology search approach and function prediction approach to classify genes of 11 Euplotia species and eight Hypotrichia species. We processed homology search approach using OrthoFinder and obtained 290,432 homologous groups. Of all the homologous groups, 1426 were presented in all species and seven were single-copy homologous groups. InterProScan [27] was used to identify conserved functional 
protein domains of genes in the 19 species. We finally obtained 5991 Pfam domains which were classified into 2897 clans. To investigate potential gene expansion events, we performed gene family gain/loss analysis to the 2897 clans using CAFE [28] and found that 234 clans showed significant gain/loss changes. The number of gain/loss genes was 628/411 in Diophrys, 596/281 in Uronychia, 365/1344 in Certesia, and 122/334 in Euplotes (Additional file 1: Fig. S6). By comparing Euplotia with Hypotrichia using Fisher's exact test, 39 clans showed a significant discrepancy between the two subclasses $(P<0.01)$. Clans correlated to motor protein genes, tubulin genes, and tubulin modification genes were found to be significantly expanded in Euplotia (Fig. 3A). Motor protein genes and tubulin genes are closely associated with the ciliary movement in ciliates [29], which may imply Euplotia might have a better movement ability than Hypotrichia. In addition to genes related to ciliary movement, Euplotia species also underwent expansion in genes related to environmental adaptation, such as $\mathrm{C} 2 \mathrm{H} 2$ zinc finger proteins, cytochrome P450, ATP-grasp gene, and ABC membrane (Fig. 3B).

To identify the relationship between gene expansion and speciation, we compared the results of homology search approach using OrthoFinder and function prediction approach using InterProScan. Among the 5991 annotated Pfam domains, 937 (15.6\%) contain only one homologous group, and 585 (9.8\%) contain more than five homologous groups. Among the 39 clans with a significant discrepancy between Euplotia and Hypotrichia, the cytochrome P450 clan contains 57 homologous

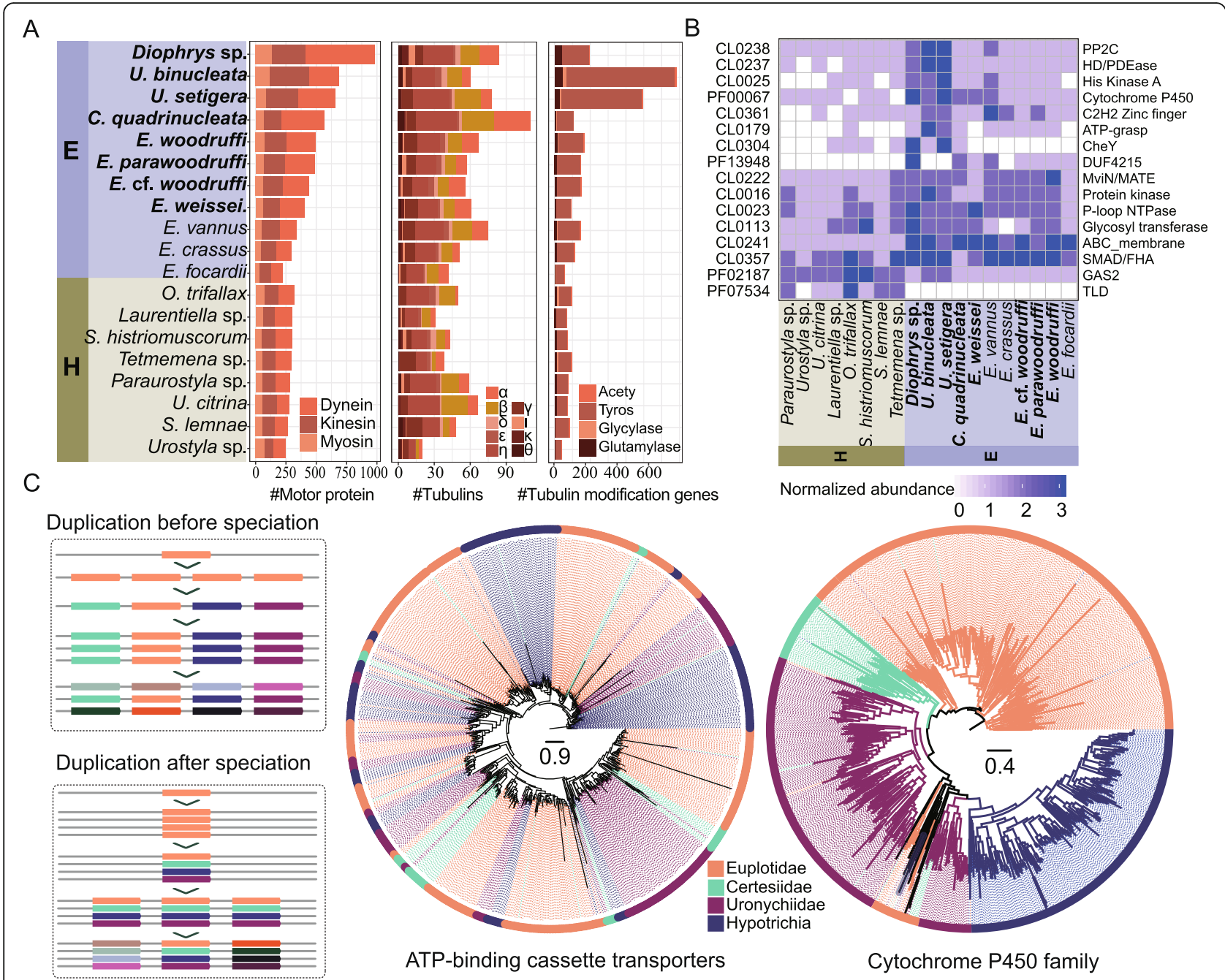

Fig. 3 Extensive gene amplification events in Euplotia. A The counts of genes related to cilium kinetics in each species of Euplotia and Hypotrichia. B Significantly expanded/contracted gene families among all species of Euplotia and Hypotrichia. Each cell in the heatmap represents the normalized gene count of a Pfam family. $\mathbf{C}$ Gene duplication before and after speciation. The phylogenetic trees on the right side were constructed based on ABC transporters and Cytochrome P450 gene families using the ML method, respectively 
groups and ATP-binding cassette transporters contain 42 homologous groups. To find out the relationship between these genes and speciation, we performed phylogenetic analysis of $\mathrm{ABC}$ transporter genes and cytochrome P450 genes. As shown in Fig. 3C, cytochrome $\mathrm{P} 450$ genes and $\mathrm{ABC}$ transporter represent two different modes of gene duplication. ABC transporterrelated genes were divided into several subgroups, and genes from each species were dispersed in each group, indicating that most of these genes were amplified before speciation. In contrast, cytochrome $\mathrm{P} 450$ genes were classified into four subgroups consistent with the taxonomic classification, indicating that they may have been duplicated after speciation.

\section{Four distinct subgroups in genus Euplotes}

As the largest and most diverse genus of ciliates, Euplotes exhibits many genus-specific characteristics, such as unique genetic codons, a high frequency of programmed ribosomal frameshifts, well-developed AZMs, and a single undulating membrane $[4,21]$. The genomic divergence of different species of Euplotes is still unknown because of the lack of genomic data. Here, the successful assembly of four new genomes in this genus enables a comprehensive comparison of genome structure and gene content among the different species. We first performed a synteny analysis of the seven Euplotes genomes and found that they could be divided into four subgroups based on their syntenic similarities (Fig. 4A). E. cf. woodruffi, E. woodruffi, and E. parawoodruffi formed one subgroup with an average of 512 syntenic regions. E. vannus and M. crassus formed another subgroup that shared 307 syntenic regions. The genomic syntenies of E. focardii and E. weissei were distinct from those of any other species and formed two separate subgroups.

We annotated genes in the same syntenic regions and found that these genes were generally functionally associated. For example, cyclic nucleotide phosphodiesterase (PDEase_I) and ion transporter (Ion_trans) were always adjacent to each other among the four subgroups despite their limited sequence identities (Fig. 4B). Previous studies found that cyclic nucleotide phosphodiesterase can regulate the activity of ion transport proteins by controlling cAMP or cGMP in cyclic nucleotide-gated ion channels [30]. Meanwhile, tandem or segmental duplication events (e.g., kinesin) may also contribute to the divergence of gene synteny between different subgroups (Fig. 4B).

To determine the phylogenetic relationships among the four subgroups, we first constructed a phylogenetic tree using 157 ortholog genes. The intrasubgroup phylogenetic distances were generally less than 0.01 , which was significantly lower than those between subgroups (>
0.1) $(t$ test, $P<0.01)$ (Fig. 4C). Then, we calculated the pairwise protein identity for each homologous group across different species. The number of shared homologous group and their protein identities within each subgroup were higher than those between subgroups. For example, subgroup 1 and subgroup 2 had 9785 and 8966 homologous genes, respectively, with an average amino acid identity $>95 \%$. In contrast, Euplotes spp. had an average of 5663 shared genes between subgroups, with an average amino acid identity $<60 \%$ (Fig. 4D). We further used Fisher's exact test to find significantly enriched or depleted Pfam domains among these subgroups. As shown in Fig. 4E, most of these Pfam domains/clans were involved in sulfur and ammonium metabolism and transport, which may be associated with environmental adaptations.

\section{Genomic characters of the newly sequenced genus Uronychia}

Uronychia is a cosmopolitan ciliated organism that inhabits marine environments or saltwater. Although there are numerous studies concerning its morphology, morphogenesis, and taxonomy, the genus Uronychia remains one of the most mysterious ciliates due to the lack of whole-genome or transcriptome data [12, 31]. Compared to other species in Spirotricha, Uronychia has more rigid and sculptured body extracellular matrices, which makes its cell very stable. Meanwhile, Uronychia swims faster than other species and has rarely seen sudden jump behavior [2, 4, 32]. These characteristics indicate that Uronychia may have unique genome features related to cell wall generation and ciliary movement.

To explore the genetic divergence between the genus Uronychia and its relatives in Euplotia and Hypotrichia, we first compared the genomic synteny of two Uronychia species to the seven Euplotes and 10 Hypotrichia species we described above. The genomic structure between $U$. setigera and $U$. binucleate was highly conserved but divergent from that of other species. We further analyzed the genes shared in these species and their protein similarity. The number of homologous proteins and their protein identity between the two Uronychia is higher than that of other species. For example, U. setigera has 172 syntenic regions with $U$. binucleate, but only has five syntenic regions with E. parawoodruffi and five syntenic regions with $O$. trifallax (Fig. 5A). Meanwhile, protein identity of homologous genes between the two Uronychia species (63\%) is higher than the average identity of $U$. setigera, $U$. binucleate, E. parawoodruffi, and O. trifallax (40\%) (Fig. 5B).

Next, we performed Gene Ontology (GO) enrichment analysis for the two Uronychia species. Nine thousand eight hundred seventy-four genes of $U$. binucleate were classified into $711 \mathrm{GO}$ terms, and 9838 genes of $U$. 


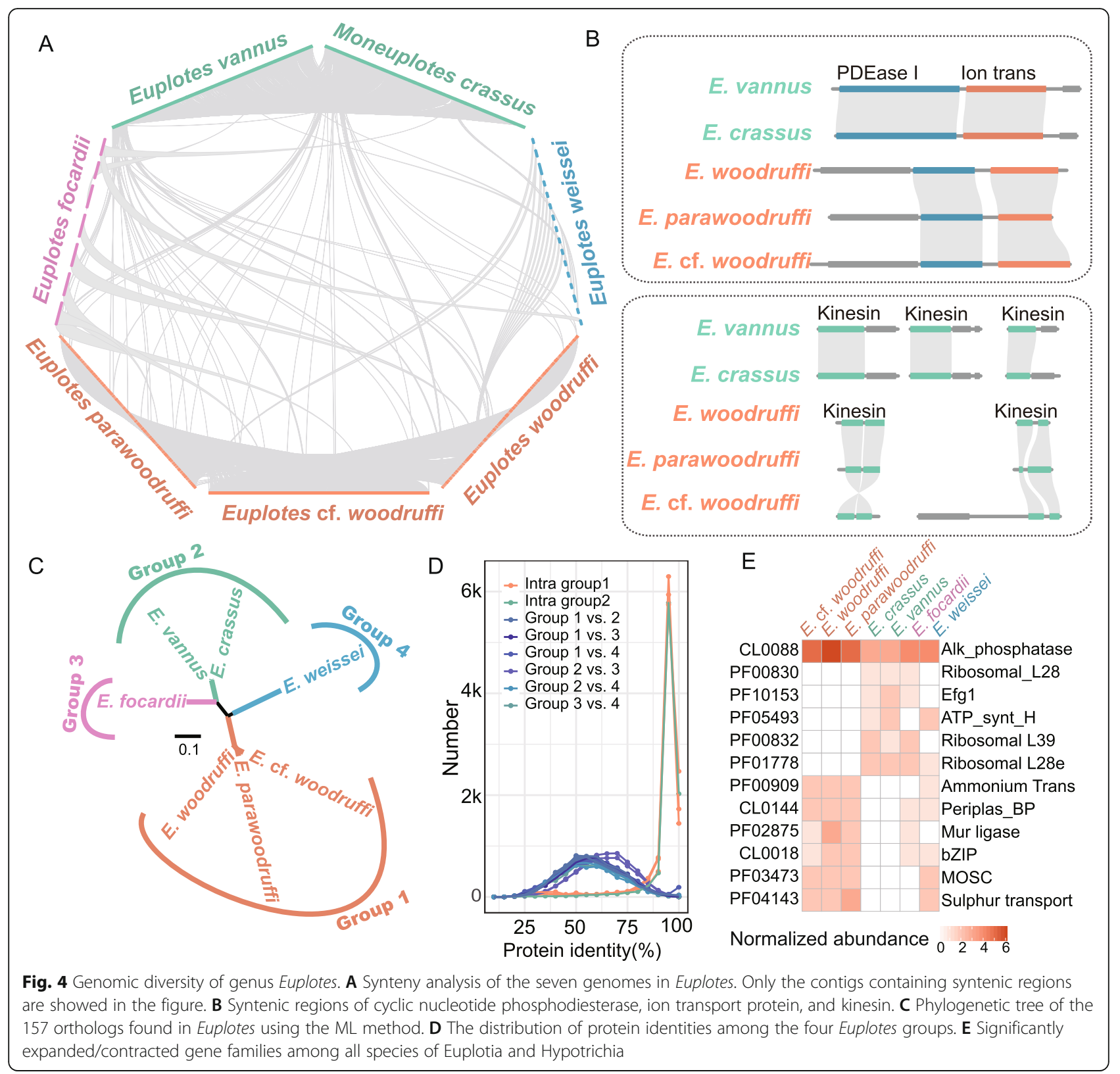

setigera were classified into 777 terms. We compared the enriched GO terms between Uronychia species and other 22 Spirotricha species using Fisher's exact test and found 109 terms were significantly different $(P<0.01)$, including terms related to chitin synthesis, microtubule, signal transduction, sulfur metabolism, and carboxypeptidase. Chitin is an important component of ciliates [33], and there is a significant gene expansion of chitin synthesis gene in Uronychia, which is consistent with the morphological structure of Uronychia. Genes related to carboxypeptidase in Uronychia are six times higher than those in other Spirotricha species, indicating Uronychia may have diverse functions in protein degradation. Both adenylate cyclase genes and phospholipase genes were significantly expanded, which may suggest that Uronychia has a strong regulation of cyclic adenosine monophosphate (cAMP). In addition, genes related to transsulfuration, vacuolar transport, lipid catabolic, and antioxidant activity underwent contraction and genes related to ion transport and signal transduction underwent expansion (Fig. 5C).

We further proposed a model of ciliary movement machinery based on comparative genomic analysis (Fig. 5D). The axoneme, made up of nine doublet microtubules, is the scaffold of the cilia and provides tracks for molecular motors [34]. Dynein and kinesin on the axoneme move cilia through the intraflagellar transport process [35], in which ion transport proteins may 


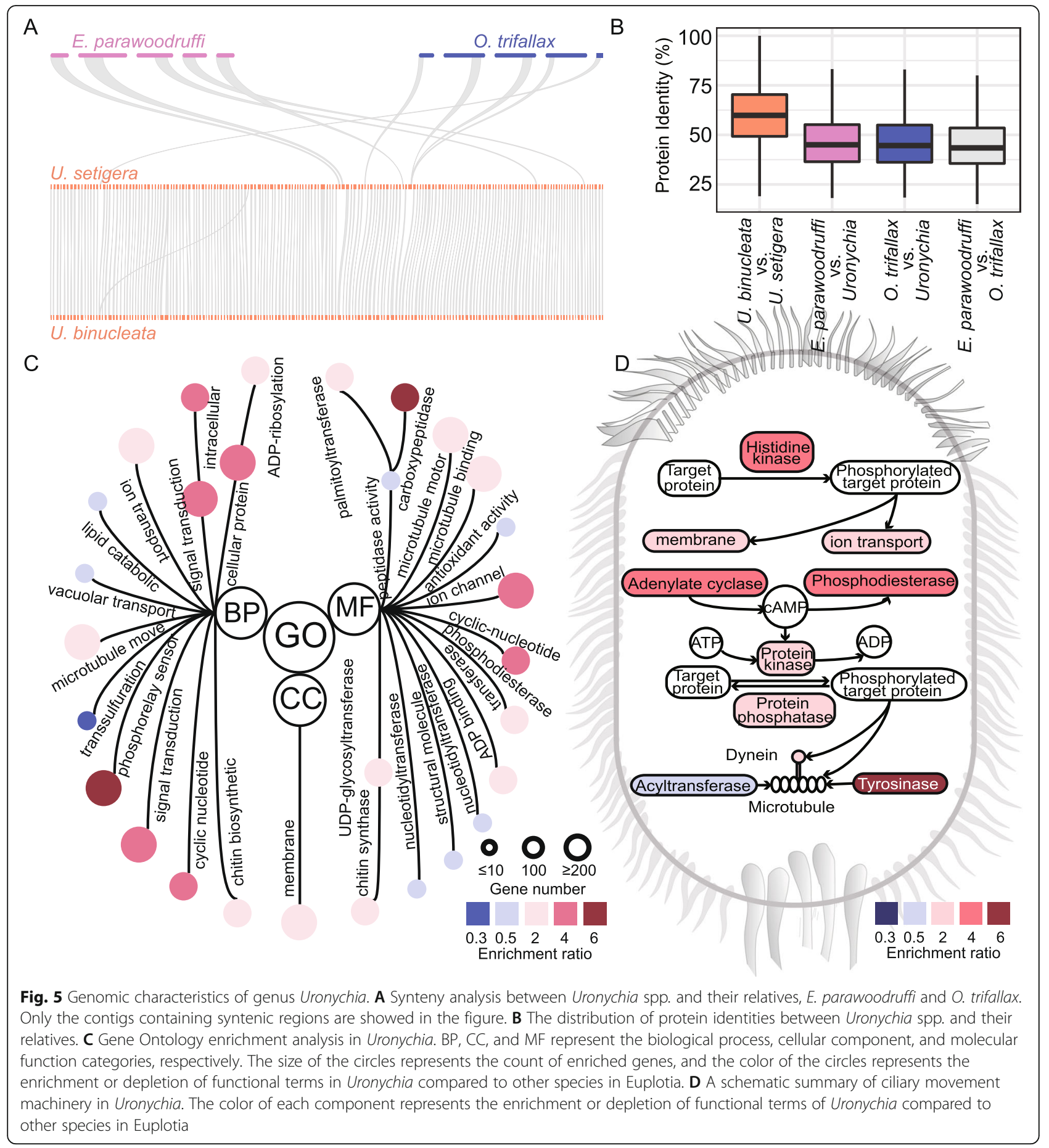

provide energy for ciliary movement [36]. Cyclase and phosphodiesterase regulate the activity of ion transport proteins, dynein and the membrane by controlling the level of cAMP [37, 38], which is an important second messenger, to protein kinases by activating ciliumrelated proteins. As shown in Fig. 5D, Uronychia exhibited a significant expansion in adenylate cyclase, phosphodiesterase, protein kinase, and ion transporter, which may explain its unique and strong swimming ability.

\section{Discussion}

As one of the most diverse ciliate subclass [4], the study of the genome diversity of Euplotia is important for understanding its phylogenetic history and environmental adaptation. Currently, only three species in this subclass 
have published genomes, and more genomic information from other genera is needed to study their genomic diversity. Although there have been some applications of single-cell sequencing in ciliates $[9,10,39]$, the sequencing and analysis methods they used are not sufficient to obtain complete genomes, thus hindering the study of genomic diversity in ciliates. In this study, we optimized the single-cell sequencing methods and corresponding data analyses to effectively remove contamination by commensal bacteria and to generate high-quality ciliate genomes. We obtained eight ciliate genomes using this approach, and through comparative genomics analysis, we characterized the underestimated genomic diversity of Euplotia and determined the divergence time of representative species in this subclass for the first time; we also further investigated the extensive duplication events associated with speciation and environmental adaptation.

Single-cell sequencing has been applied to explore ciliate genomes and transcriptomes. Due to insufficient DNA content and high amplification bias, it is impossible to obtain a complete genome from a single cell. In addition, amplification of too many cells may increase the error rate caused by single-cell amplification and genomic differences between cells. By combining the advantages of MDA and MALBAC, we successfully obtained both better genome coverage and less amplification bias when processing the DNA samples of these uncultured ciliate cells. The incorporation of whole-genome sequence from a few more cells (2 4) can improve the final genome assembly without sacrificing homogeneity. In addition, excluding bacterial contamination is an important challenge in nonmodel organism genomics research. In this study, we found that the percentage of bacteria-derived reads in the raw sequencing data was generally below $10 \%$, which was significantly lower than that in bulk whole-genome sequencing. Using the iterative filtration method, we were able to remove most of the nonciliate contaminants and obtain eight high-quality Euplotia genomes.

Through the combination of homology search approach and functional annotation approach, we found that genes related to environmental adaption exhibited huge expansion. When a gene duplication event occurs after speciation, the genes tend to vary greatly between species due to evolution. In contrast, when gene duplication events occur before speciation, the genes tend to divide into several lineages shared by all species (Fig. $3 C)$. In highly expanded genes that are related to environmental adaption, we observed the relationship between gene duplication and speciation. This finding provides some insights into the correlation between speciation and environmental adaption. Through comparative analysis, we found that the diversity and complexity of Euplotia genomes were higher than expected. The genus Euplotes contains more than 80 species, most of which have similar cell shapes, cirri counts, and welldeveloped AZMs [4]. Based on an analysis of the assembled genomes from single-cell sequencing, we found that these seven species should be divided into four subgroups. In addition to the diversity of Euplotia, we further found that Euplotia exhibited chitin synthase gene expansion compared to Hypotrichia, which may be responsible for the more rigid lorica structure in Euplotia. This study demonstrates the power of single-cell genome sequencing in investigating the genomic diversity and novel characteristics of unculturable ciliates. The eight high-quality genomes obtained in this study will help understand the genetic basis of ciliate evolution and phenotypic adaptation.

\section{Conclusions}

In this study, we optimized the single-cell sequencing methods and corresponding data analyses to effectively remove contamination by commensal bacteria and to generate high-quality ciliate genomes. We obtained eight ciliate genomes using this approach, and through comparative genomics analysis, we characterized the underestimated genomic diversity of Euplotia and determined the divergence time of representative species in this subclass for the first time. We further investigated the extensive duplication events associated with speciation and environmental adaptation. This study provides a unique and valuable resource for understanding the evolutionary history and genetic diversity of ciliates.

\section{Methods}

Sample collection and genome sequencing

E. woodruffi was collected from a freshwater pond in Taipingjiao Park in Qingdao, China. E. cf. woodruffi was collected from 17\% brackish water pond in Shenzhen Binhai Park, China. E. parawoodruffi was collected from $15 \%$ brackish water from Qingdao Coast, China. The other samples were collected from coastal seawater in Qingdao, China (Fig. 1B). All samples were poured into Petri dishes, and ciliate cells were isolated using glass pipettes. For microscopic inspection, the samples were silver stained to reveal their infraciliature. After morphological observation, the samples were washed 35 times to remove contaminants, and the genomic DNA was amplified using the REPLI-g Single Cell Kit (Qiagen) and the Single-Cell WGA Kit (Yikon, YK001A). For each species, two cells were subjected to each amplification method according to the manufacturer's guidelines. Sequencing libraries were constructed using the TruSeq Nano DNA HT Sample Preparation Kit (Illumina). High-throughput sequencing was performed on an Illumina NovaSeq 6000 platform with PE150. 


\section{Genome assembly}

Sequencing data from the same species were evaluated using FastQC v0.11.9 (http://www.bioinformatics. babraham.ac.uk/projects/fastqc/) and merged. Fastp v0.20.1 [40] was used to trim adapters and polymers with default parameters. Low-quality reads were filtered with Trimmomatic 0.39 [41] (LEADING:20 TRAILING: 20 SLIDINGWINDOW:4:25 MINLEN:120 AVGQUAL: 28). Spades v3.13.0 [42] was used to assemble pairedend reads into contigs with single-cell option and multikmer options (-k 61,71, 77, 81). Then, the following procedures were used to remove potential contamination: (1) MetaGeneMark v3.38 [43] was used to annotate assembled contigs; (2) gene fragments assigned to bacterial or algal species by searching against the NR database using DIAMOND v0.9.26 [44] were considered contaminants; and (3) Bowtie 2.4.1 [45] was used to align paired-end reads against the target bacterial genomes downloaded from the NCBI RefSeq database. Transcriptome datasets of Euplotia downloaded from the NCBI SRA database were assembled using Trinity 2.8.5 [46] with default parameters. QUAST v5.0.2 [47] with default parameters was used to determine the statistics of the assembled genomes. EukCC v0.2 [19] with default parameters was used to assess genome completeness at the protein level. BUSCO v4.0.6 [20] was used to assess the assembly quality using Alveolata dataset and protein mode.

\section{Gene prediction and annotation}

AUGUSTUS v3.3.3 [48] was used to predict genes from assembled contigs. The eight species we sequenced used two nonstandard codons to translate proteins. The species Certesia quadrinucleata, Diophrys sp., Uronychia binucleate, and Uronychia setigera used "translation table 6" (https://www.ncbi.nlm.nih.gov/Taxonomy/Utils/ wprintgc.cgi, TAA/TAG encodes glutamine compared to the standard code) as its codon, which is the same as Tetrahymena. The other four species, Euplotes cf. woodruffi, Euplotes parawoodruffi, Euplotes weissei, and Euplotes woodruffi, used "translation table 10" (TGA encodes cystine). For species using translation table 6, we used the Tetrahymena dataset to predict genes. For species using translation table 10, we modified the configuration file of the Tetrahymena dataset by choosing translation table 10, and then performed gene prediction. To ensure the quality of the predicted genes, we also trained AUGUSTUS by using nucleic acid and protein sequences of Oxytricha trifallax (http://oxy.ciliate. org/index.php/home/downloads) and Euplotes vannus (http://evan.ciliate.org/index.php/home/downloads). The NR database was used to annotate genes using DIAMOND v0.9.26 in protein search mode $(E$ value $<1 \mathrm{E}$ $-5)$. Motifs and protein domains were annotated against the PANTHER, Pfam, Gene3D, and CDD databases using InterProScan v10.0.2 [27]. GO and KEGG annotations for each gene were extracted from the corresponding InterPro entries.

\section{Gene family analysis}

OrthoFinder v2.3.12 [26] was applied to find homologs among 24 Spirotricha species. In the OrthoFinder process, DIAMOND v0.9.26 was used to search sequence, MAFFT v7.470 [49] was used to align multiple sequences, and FastTree 1.0 [50] was used to infer phylogenetic tree. The homologous groups were clustered using k-means algorithm based on their gene abundance in each species. The protein identity for each homologous group between every species was calculated using a custom Perl script. All homologous groups were assigned to different Pfam domains based on the annotated results above. CAFE v4.2.1 [28] was used to compute gene expansion and contraction for the homologous groups between every species.

\section{Phylogenetic analysis and divergence time estimation}

We used two different multiple alignment datasets for phylogenetic analysis. The first dataset contained singlecopy genes of 24 Spirotricha species using protein by protein search process in OrthoFinder. The second dataset was obtained from GPSit v1.0 [22] pipeline by searching 157 orthologous genes in the 24 species. The sequences were trimmed using PREQUAL v1.02 [51] with threshold zero, aligned using MUSCLE v3.8.31 [52] with default parameters, retrimmed using Divvier v1.01 [53] with default parameters. After trimming and alignment, proteins of those two datasets were concatenated, respectively. BMGE v1.12 [54] was used to mask ambiguous sites of the alignments. IQ-Tree v1.6.12 [55] was used to construct the ML trees with $\mathrm{C} 60+\mathrm{LG}+\mathrm{G}+\mathrm{F}$ parameters. BEAST v1.10.4 [25] was used to construct the time-scaled trees under JTT substitution model and Strict clock model. Markov chain Monte Carlo (MCMC) sample chains were run for $10^{7}$. The convergence of parameters was evaluated using Tracer v1.7.1 [56].

\section{Genome synteny analysis}

JCVI utility libraries v1.0.9 [57] were used to analyze and visualize the synteny between different genomes. LASTAL was used to perform pairwise synteny search with C-score cutoff of 0.7. MCScanX v1.0 [58] was used to detect syntenic regions with a cutoff of three genes. Genes in the same synteny region were extracted using custom Perl scripts and annotated using InterProScan v10.0.2 to determine the relationship between genome synteny and gene functions. 


\section{Supplementary Information}

The online version contains supplementary material available at https://doi. org/10.1186/s12915-021-01202-1

Additional file 1: Tables S1-S3, Figures S1-S6. Supplementary tables and figures. Table S1. Single-cell amplification of eight Ciliates. Table on MDA and MALBAC methods for amplification of single cells. "1" means single-cell amplification success, "0" means amplification failure. For each amplification experiment, only one cell was used. Table S2. Information of 24 ciliate genomes. Table summarizing the data category, data source, accession number and hyperlink of the species used in this research.

Table S3. Commands for analyzing process. Software, versions and parameters used for each analysis step. Fig. S1. Genome completeness assessed by BUSCO and EukCC. The completeness of the eleven Euplotia genomes assessed using EukCC and BUSCO software. Bold font indicates the species newly sequenced in this research, and regular font indicates species already published. Fig. S2. Percentage of contigs containing the number of genes. The proportion of contigs containing different gene counts out of the total contigs in each genome. Contigs containing more than 5 genes were grouped together. Fig. S3. Phylogenetic tree of all the sequenced ciliate species. Phylogenetic tree based on 157 gene sequences. The tree was constructed by the maximum likelihood method ( $660+\mathrm{LG}+\mathrm{G}+\mathrm{F}$ model). The numbers on the nodes represent the bootstrap values. Fig. S4. Phylogenetic tree based on single-copy genes. Phylogenetic tree of 19 Euplotia species base on single-copy genes obtained from compared genetic analysis. Fig. S5. Gene ontology enrichment. Gene ontology enrichment of housekeeping genes in Euplotia. The tree was constructed by the maximum likelihood method ( $60+\mathrm{LG}+\mathrm{G}+\mathrm{F}$ model). The numbers on the nodes represent the bootstrap values. Fig. S6. Gene gain/loss in this study. Gene gain/loss in Euplotia and Hypotrichia. Red letter represents gene gain, and blue letter represents gene loss.

\section{Acknowledgements}

We thank Prof. Weibo Song (Ocean University of China) for the guidance on the experiment.

\section{Authors' contributions}

M.M. conceived the project. W.C., C.Z., and F.Z. performed the data analysis. C. W. and T. Z. collected ciliates samples. L.L. and Y.Q. performed the experiments and generated sequencing data. W.C., F.Z., and M.M. wrote the manuscript with the contribution of all authors. All authors read and approved the final manuscript.

\section{Funding}

This work was supported by grants from National Natural Science Foundation of China [32070432, 32025009, 31672279 and 31671364] and the Fundamental Research Funds for the Central Universities.

\section{Availability of data and materials}

All data generated or analyzed during this study are included in this published article and its supplementary information files and publicly available repositories. Reads for the genome assemblies have been deposited in GenBank's Short Read Archive (SRA) under BioProject number PRJNA721552. The software and parameters for each analysis step were listed in Additional file 1: Table S3. The single-gene alignments, the output of comparative genomics statistics, single copy homologous genes, and tables for drawing figures of this study are available at the Figshare website with DOl: https://doi.org/10.6084/m9.figshare.17078357. The custom scripts are available at the website Github: https://github.com/trainrun/Euplotes_script.

\section{Declarations}

Ethics approval and consent to participate Not applicable.

\section{Consent for publication}

Not applicable.

\section{Competing interests}

The authors declare that they have no competing interests.

\section{Author details}

${ }^{1}$ Savaid Medical School, University of Chinese Academy of Sciences, Beijing 100049, China. ${ }^{2}$ Beijing Institutes of Life Science, Chinese Academy of Sciences, Beijing 100101, China. ${ }^{3}$ Institute of Evolution \& Marine Biodiversity, Ocean University of China, Qingdao 266003, China. ${ }^{4}$ Key Laboratory of Mariculture (OUC), Ministry of Education, Qingdao 266003, China.

Received: 10 February 2021 Accepted: 1 December 2021

Published online: 14 December 2021

\section{References}

1. Foissner W, Chao A, Katz LA. Protist diversity and geographical distribution. Dordrecht: Springer Netherlands; 2009. https://doi.org/10.1007/978-90-481-2 801-3.

2. Lynn, Denis H. The ciliated protozoa. 2010;10.1007/97.

3. Mochizuki K. DNA rearrangements directed by non-coding RNAs in ciliates. WIREs RNA. 2010;1(3):376-87. https://doi.org/10.1002/wrna.34.

4. Song W, Warren A. Free-living ciliates in the Bohai and Yellow Seas, China. 2009.

5. Kawaida H, Ohba K, Koutake Y, Shimizu H, Tachida H, Kobayakawa Y. Symbiosis between hydra and chlorella: molecular phylogenetic analysis and experimental study provide insight into its origin and evolution. Mol Phylogenet Evol. 2013;66(3):906-14. https://doi.org/10.1016/j.ympev.2012.11. 018.

6. Ishikawa M, Yuyama I, Shimizu H, Nozawa M, Ikeo K, Gojobori T. Different endosymbiotic interactions in two hydra species reflect the evolutionary history of endosymbiosis. Genome Biol Evol. 2016;8(7):2155-63. https://doi. org/10.1093/gbe/evw142.

7. Aury J-M, Jaillon O, Duret L, Noel B, Jubin C, Porcel BM, et al. Global trends of whole-genome duplications revealed by the ciliate Paramecium tetraurelia. Nature. 2006;444(7116):171-8. https://doi.org/10.1038/nature0523 0 .

8. Eisen JA, et al. Macronuclear genome sequence of the ciliate Tetrahymena thermophila, a model eukaryote. PLoS Biol. 2006:4(9):1620-42.

9. Yan Y, Maurer-Alcalá XX, Knight R, Pond SLK, Katz LA. Single-cell transcriptomics reveal a correlation between genome architecture and gene family evolution in ciliates. MBio. 2019;10(6):1-13. https://doi.org/10.1128/ mBio.02524-19.

10. Chen X, Wang C, Pan B, Lu B, Li C, Shen Z, et al. Single-cell genomic sequencing of three peritrichs (Protista, Ciliophora) reveals less biased stop codon usage and more prevalent programmed ribosomal frameshifting than in other ciliates. Front Mar Sci. 2020;7:1-8. https://doi.org/10.3389/fma rs.2020.602323.

11. Lin X, Song W. Redescription of the marine ciliate, Certesia quadrinucleata (Protozoa: Ciliophora) from Qingdao, China J. Mar Biol Assoc United Kingdom. 2004;84(6):1131-6. https://doi.org/10.1017/S0025315404010562h.

12. Valbonesi A, Luporini P. A new species of Uronychia (Ciliophora, Hypotrichida) from Antarctica: Uronychia antarctica. Bolletino di Zool. 1990; 57(4):365-8. https://doi.org/10.1080/11250009009355721.

13. Press P, Der Trichocysten B, Sons JWC. Morphological comparison of Diobhrys scutum (Dujardin, 1841) and Diophrys peloetes n. sp. (Hypotrichida, Ciliophora). 1963;31:60-6.

14. Curds CR. A guide to the species of the genus Euplotes (Hypotrichida, Ciliatea). Bull Br Museum His. 1975;28.

15. Song W, et al. Redescriptions of two marine hypotrichous ciliates, Diophrys irmgard and D. hystrix (Ciliophora, Euplotida), with a brief revision of the genus Diophrys. J Eukaryot Microbiol. 2010;54(3):283-96. https://doi.org/1 0.1111/j.1550-7408.2007.00266.x.

16. Dean BF. Rapid amplification of plasmid and phage DNA using Phi29 DNA polymerase and multiply-primed rolling circle amplification. Genome Res. 2001;11(6):1095-9. https://doi.org/10.1101/gr.180501.

17. Lu S, Zong C, Fan W, Yang M, Li J, Chapman AR, et al. Probing meiotic recombination and aneuploidy of single sperm cells by whole-genome sequencing. Science (80-. ). 2012;338(6114):1627-30. https://doi.org/10.1126/ science.1229112

18. Wood DE, Salzberg SL. Kraken: ultrafast metagenomic sequence classification using exact alignments. Genome Biol. 2014;15(3). 
19. Saary P, Mitchell A, Finn R. Estimating the quality of eukaryotic genomes recovered from metagenomic analysis with EukCC. Genome Biol. 2019;21(1): 1-21. https://doi.org/10.1186/s13059-020-02155-4.

20. Simão FA, Waterhouse RM, loannidis $P$, Kriventseva EV, Zdobnov EM BUSCO: assessing genome assembly and annotation completeness with single-copy orthologs. Bioinformatics. 2015;31(19):3210-2. https://doi.org/1 0.1093/bioinformatics/btv351.

21. Chen X, Jiang Y, Gao F, Zheng W, Krock TJ, Stover NA, et al. Genome analyses of the new model protist Euplotes vannus focusing on genome rearrangement and resistance to environmental stressors. Mol Ecol Resour. 2019;19(5):1292-308. https://doi.org/10.1111/1755-0998.13023.

22. Chen X, Wang Y, Sheng Y, Warren A, Gao S. GPS it: an automated method for evolutionary analysis of nonculturable ciliated microeukaryotes. Mol Ecol Resour. 2018;18(3):700-13.

23. Sun $P$, Clamp J, Xu D, Huang B, Shin MK. An integrative approach to phylogeny reveals patterns of environmental distribution and novel evolutionary relationships in a major group of ciliates. Sci Rep. 2016;6:1-12.

24. Sheng Y, He M, Zhao F, Shao C, Miao M. Phylogenetic relationship analyses of complicated class Spirotrichea based on transcriptomes from three diverse microbial eukaryotes: Uroleptopsis citrina, Euplotes vannus and Protocruzia tuzeti. Mol Phylogenet Evol. 2018;129:338-45.

25. Drummond AJ, Rambaut A. BEAST: Bayesian evolutionary analysis by sampling trees. BMC Evol Biol. 2007;7(1):214

26. Emms DM, Kelly S. OrthoFinder: phylogenetic orthology inference for comparative genomics. Genome Biol. 2019;20(1):1-14. https://doi.org/10.11 86/s13059-019-1832-y.

27. Quevillon E, et al. InterProScan: protein domains identifier. Nucleic Acids Res. 2005;33(suppl_2):W116-20.

28. De Bie T, Cristianini N, Demuth JP, Hahn MW. CAFE: a computational tool for the study of gene family evolution. Bioinformatics. 2006;22(10):1269-71. https://doi.org/10.1093/bioinformatics/btl097.

29. Verhey KJ, Dishinger J, Kee HL. Kinesin motors and primary cilia. Biochem Soc Trans. 2011;39(5):1120.

30. Kaupp UB, Seifert R. Cyclic nucleotide-gated ion channels. Physiol Rev Jul. 2002;82(3):769-824. https://doi.org/10.1152/physrev.00008.2002

31. Song WB, Wilbert N, Chen ZG, Shi XL. Considerations on the systematic position of uronychia and related euplotids based on the data of ontogeny and 185 rRNA gene sequence analyses, with morphogenetic redescription of Uronychia setigera Calkins, 1902 (Ciliophora: Euplotida). Acta Protozool. 2004:43(4):313-28

32. Corliss JO. The ciliated protozoa: characterization, classification, and guide to the literature. Trans Am Microsc Soc. 1979;98(3).

33. Yang Q, Fukamizo T. Targeting chitin-containing organisms, vol. 1142. Singapore: Springer; 2019.

34. Bayless BA, Navarro FM, Winey M. Motile Cilia: innovation and insight from ciliate model organisms. Front Cell Dev Biol. 2019;7:1-8.

35. Dhanasekaran N, Reddy EP. Signaling by dual specificity kinases. Oncogene. 1998;17(11):1447-55. https://doi.org/10.1038/sj.onc.1202251

36. Scheer BT. "Ion transport." AccessScience. https://doi.org/10.1036/10978542.352000. Accessed 30 Nov 2020.

37. Bender AT. Cyclic nucleotide phosphodiesterases: molecular regulation to clinical use. Pharmacol Rev. 58(3):488-2006.

38. Zhang G, Liu Y, Ruoho AE, Hurley JH. Structure of the adenylyl cyclase catalytic core. Nature. 1997;386(6622):247-53. https://doi.org/10.1038/38624 $7 \mathrm{a} 0$

39. Pan B, Chen X, Hou L, Zhang Q, Qu Z, Warren A, et al. Comparative genomics analysis of ciliates provides insights on the evolutionary history within 'Nassophorea-Synhymenia-Phyllopharyngea' assemblage. Front Microbiol. 2019;10:1-13. https://doi.org/10.3389/fmicb.2019.02819.

40. Chen S, Zhou Y, Chen Y, Gu J. fastp: an ultra-fast all-in-one FASTQ preprocessor. Bioinformatics. 2018;34(17):i884-90. https://doi.org/10.1093/ bioinformatics/bty560.

41. Bolger $A M$, Lohse $M$, Usadel B. Trimmomatic: a flexible trimmer for Illumina sequence data. Bioinformatics. 2014;30(15):2114-20. https://doi.org/10.1093/ bioinformatics/btu170.

42. Bankevich A, Nurk S, Antipov D, Gurevich AA, Dvorkin M, Kulikov AS, et al. SPAdes: a new genome assembly algorithm and its applications to singlecell sequencing. J Comput Biol. 2012;19(5):455-77. https://doi.org/10.1089/ cmb.2012.0021.
43. Zhu W, Lomsadze A, Borodovsky M. Ab initio gene identification in metagenomic sequences. Nucleic Acids Res. 2010;38(12):e132. https://doi. org/10.1093/nar/gkq275

44. Buchfink $B$, Xie $C$, Huson DH. Fast and sensitive protein alignment using DIAMOND. Nat Methods. 2015;12(1):59-60. https://doi.org/10.1038/nmeth.31 76.

45. Langmead B, Salzberg SL. Fast gapped-read alignment with Bowtie 2. Nat Methods. 2012;9(4):357.

46. Haas BJ, Papanicolaou A, Yassour M, Grabherr M, Blood PD, Bowden J, et al. De novo transcript sequence reconstruction from RNA-seq using the Trinity platform for reference generation and analysis. Nat Protoc. 2013:8(8):1494512. https://doi.org/10.1038/nprot.2013.084.

47. Gurevich A, Saveliev V, Vyahhi N, Tesler G. QUAST: quality assessment tool for genome assemblies. Bioinformatics. 2013;29(8):1072-5. https://doi.org/1 0.1093/bioinformatics/btt086

48. Stanke M, Keller O, Gunduz I, Hayes A, Waack S, Morgenstern B. AUGUSTUS: ab initio prediction of alternative transcripts. Nucleic Acids Res. 2006; 34(suppl_2):W435-39.

49. Katoh K, Misawa K, Kuma Kl, Miyata T. MAFFT: a novel method for rapid multiple sequence alignment based on fast Fourier transform. Nucleic Acids Res. 2002;30(14):3059-66. https://doi.org/10.1093/nar/gkf436.

50. Price MN, Dehal PS, Arkin AP. Fasttree: Computing large minimum evolution trees with profiles instead of a distance matrix. Mol Biol Evol. 2009;26(7): 1641-50. https://doi.org/10.1093/molbev/msp077.

51. Whelan S, Irisarri I, Burki F. PREQUAL: Detecting non-homologous characters in sets of unaligned homologous sequences. Bioinformatics. 2018;34(22): 3929-30.

52. Edgar RC. MUSCLE: A multiple sequence alignment method with reduced time and space complexity. BMC Bioinformatics. 2004;5:1-19.

53. Ali RH, Bogusz M, Whelan S. "A graph-based approach for improving the homology inference in multiple sequence alignments." [Preprint] 2018. Available from: https://www.diva-portal.org/smash/record.jsf?pid= diva2\%3A1249291.

54. Criscuolo A, Gribaldo S. BMGE (Block Mapping and Gathering with Entropy): a new software for selection of phylogenetic informative regions from multiple sequence alignments. BMC Evol Biol. 2010;10(1):210.

55. Nguyen LT, Schmidt HA, Von Haeseler A, Minh BQ. IQ-TREE: a fast and effective stochastic algorithm for estimating maximum-likelihood phylogenies. Mol Biol Evol. 2015;32(1):268-74. https://doi.org/10.1093/ molbev/msu300.

56. Rambaut A, Drummond AJ, Xie D, Baele G, Suchard MA. Posterior summarization in Bayesian phylogenetics using Tracer 1.7. Syst Biol. 2018; 67(5):901-4. https://doi.org/10.1093/sysbio/syy032.

57. Tang H, Krishnakumar V, Li J, Zhang X. jcvi: JCVI utility libraries. Zenodo. 2015. https://doi.org/10.5281/zenodo.31631.

58. Wang Y, Tang H, DeBarry JD, Tan X, Li J, Wang X, et al. MCScanX: a toolkit for detection and evolutionary analysis of gene synteny and collinearity. Nucleic Acids Res. 2012:40(7):1-14. https://doi.org/10.1093/nar/gkr1293.

\section{Publisher's Note}

Springer Nature remains neutral with regard to jurisdictional claims in published maps and institutional affiliations.

Ready to submit your research? Choose BMC and benefit from:

- fast, convenient online submission

- thorough peer review by experienced researchers in your field

- rapid publication on acceptance

- support for research data, including large and complex data types

- gold Open Access which fosters wider collaboration and increased citations

- maximum visibility for your research: over $100 \mathrm{M}$ website views per year

At $\mathrm{BMC}$, research is always in progress.

Learn more biomedcentral.com/submissions 